\title{
Article \\ Two-Colour Sum-Frequency Generation Spectroscopy Coupled to Plasmonics with the CLIO Free Electron Laser
}

\author{
Christophe Humbert ${ }^{1, *(\mathbb{D})}$, Olivier Pluchery ${ }^{2} \mathbb{D}$, Emmanuelle Lacaze ${ }^{2}$, Bertrand Busson ${ }^{1}$ \\ and Abderrahmane Tadjeddine ${ }^{1}$
}

1 Université Paris-Saclay, CNRS, Institut de Chimie Physique, UMR8000, 91405 Orsay, France; bertrand.busson@universite-paris-saclay.fr (B.B.); abderrahmane.tadjeddine@universite-paris-saclay.fr (A.T.) 2 Sorbonne Université, CNRS, Institut des NanoSciences de Paris (INSP), 4 Place Jussieu, 75005 Paris, France; olivier.pluchery@insp.jussieu.fr (O.P.); emmanuelle.lacaze@insp.jussieu.fr (E.L.)

* Correspondence: christophe.humbert@universite-paris-saclay.fr

check for updates

Citation: Humbert, C.; Pluchery, O.; Lacaze, E.; Busson, B.; Tadjeddine, A. Two-Colour Sum-Frequency Generation Spectroscopy Coupled to Plasmonics with the CLIO Free

Electron Laser. Photonics 2022, 9, 55 https://doi.org/10.3390/ photonics 9020055

Received: 22 December 2021

Accepted: 17 January 2022

Published: 20 January 2022

Publisher's Note: MDPI stays neutral with regard to jurisdictional claims in published maps and institutional affiliations.

Copyright: (C) 2022 by the authors. Licensee MDPI, Basel, Switzerland. This article is an open access article distributed under the terms and conditions of the Creative Commons Attribution (CC BY) license (https:// creativecommons.org/licenses/by/ $4.0 /)$.

\begin{abstract}
Nonlinear plasmonics requires the use of high-intensity laser sources in the visible and near/mid-infrared spectral ranges to characterise the potential enhancement of the vibrational fingerprint of chemically functionalised nanostructured interfaces aimed at improving the molecular detection threshold in nanosensors. We used Two-Colour Sum-Frequency Generation (2C-SFG) nonlinear optical spectroscopy coupled to the European CLIO Free Electron Laser in order to highlight an energy transfer in organic and inorganic interfaces built on a silicon substrate. We evidence that a molecular pollutant, such as thiophenol molecules adsorbed on small gold metal nanospheres grafted on silicon, was detected at the monolayer scale in the $10 \mu \mathrm{m}$ infrared spectral range, with increasing SFG intensity of three specific phenyl ring vibration modes reaching two magnitude orders from blue to green-yellow excitation wavelengths. This observation is related to a strong plasmonic coupling to the thiophenol molecules vibrations. The high level of gold nanospheres aggregation on the substrate allows us to dramatically increase the presence of hotspots, revealing collective plasmon modes based on strong local electric fields between the gold nanoparticles packed in close contact on the substrate. This configuration favors detection of Raman active vibration modes, for which 2C-SFG spectroscopy is particularly efficient in this unusual infrared spectral range.
\end{abstract}

Keywords: gold; nanoparticles; thiophenol; silicon; nonlinear optics; sum-frequency generation; plasmonics; UV-vis spectroscopy; atomic force microscopy; CLIO free electron laser

\section{Introduction}

Nonlinear optical Two-colour Sum-Frequency Generation (2C-SFG) spectroscopy of metal [1-3] and semiconducting [4-6] nanoparticles constitutes a reliable probe of the surface molecular chemistry of nanostructured samples in catalysis and (bio)chemical sensing [7]. For instance, in such systems, the evolution of various physico-chemical properties impacted by surface plasmons and excitons can be analysed [8,9]. In a general way, whatever the probed scale, 2C-SFG spectroscopy gives access to intramolecular vibronic couplings [10-12], molecule/substrate interactions [13-16] and molecule/nanostructure interactions [17-26]. Similar to most of the spectroscopy techniques from the nonlinear optics family [27], 2C-SFG spectroscopy works only with high-intensity laser sources. Here we need two high-intensity laser sources in the infrared (IR) and in the visible spectral ranges. While it is now possible from almost 20 years $[28,29]$ to take profit from a visible wavelength tunable from violet to red in such spectroscopy, highlighting the effect of electronic properties of any kind of interface, the major drawback was the access of the infrared wavelength in optical parametric oscillators/amplifiers (OPO/OPA). Indeed, it is quite difficult to extract enough power from nonlinear crystals (e.g., $\mathrm{AgGaS}$ ) beyond $8 \mu \mathrm{m}$ without quick damage. As a consequence, the great majority of the SFG spectroscopy literature lies in the $2.5-8 \mu \mathrm{m}$ spectral range. Vibration modes from $\mathrm{OH}, \mathrm{NH}, \mathrm{CH}, \mathrm{CN}$, 
CO chemical bonds lie in this spectral range and were extensively scrutinised with SFG. Nevertheless, probing C-C bonds around $10 \mu \mathrm{m}$, such as in phenyl rings (as in thiophenol molecules, for instance, in the present work), is highly challenging, although they are fundamental markers as evidenced in Raman spectroscopy [30] and for modelling by DFT calculations [31]. This will be our topic here.

In order to address these issues, technical improvements have been carried out for years in SFG spectroscopy by switching the pulse duration from the ps temporal regime to the fs one, in order to broaden-as much as possible in one high-intensity laser pulse-the infrared spectral range. While the fs scale allows us to reach the $10 \mu \mathrm{m}$ range, the delivered power remains limited for applications and, generally, to the detriment of the visible counterpart, limited to a fixed visible wavelength in the ps scale. While this promising approach is the most relevant for the next future of 2-Dimensional (2D) nonlinear spectroscopy for any kind of application [32], giving access to dynamical processes of surface chemistry, a successful alternative technical route for 2C-SFG spectroscopy was investigated from the beginning: the use of a high-intensity tunable infrared beam provided by Free Electron Lasers (FEL) in catalysis and electrochemistry [33-35], leading more recently to an experimental breakthrough by the SFG imaging of surface phonons [36].

In the present work, thanks to the use of the CLIO FEL [37], we successfully performed 2C-SFG spectroscopy in the $10 \mu \mathrm{m}$ IR spectral range on small gold AuNPs functionalised by thiophenol molecules, acting as a molecular surface probe, for different visible wavelengths from blue to red. In this way, we highlighted and quantified an amplification of the SFG signal of three specific thiophenol vibration modes due to an optical coupling to surface plasmon resonance. This coupling effect is related to the presence of numerous hotspots due to the strong aggregation of AuNPs. This close-packed AuNPs configuration on the substrate induces the existence of strong local electric fields between them, leading to the presence of collective plasmon modes in the yellow-orange spectral range, overwhelming the LSPR (Localised Surface Plasmon Resonance) contribution of isolated AuNPs in the green. This framework leads to the amplification of the thiophenol nonlinear vibrational response by two orders of magnitude by switching from blue $(480 \mathrm{~nm})$ to yellow-orange (560-600 nm). To the best of the authors' knowledge, this is the first time that such strong optical plasmonic coupling has been observed and calculated in this vibrational fingerprint spectral range $\left(\sim 1000 \mathrm{~cm}^{-1}\right)$ on nanostructured interface with $2 \mathrm{C}-\mathrm{SFG}$ spectroscopy.

\section{Materials and Methods}

\subsection{Sample Preparation}

The sample preparation is based on the same reliable and easily reproducible protocol described in our previous publications [38-40] as summarised hereafter and sketched in Figure 1, the purpose being for this specific study to lead to a large number of closepacked AuNPs. All chemicals were purchased from Sigma-Aldrich (France). AuNPs were synthesised from $1.7 \mathrm{mg}$ of gold salts $\left(\mathrm{HAuCl}_{4} .3 \mathrm{H}_{2} \mathrm{O}, 99.999 \%\right)$ dissolved in $20 \mathrm{~mL}$ Millipore water following Turkevich protocol. After the solution was heated up until boiling point and stirred vigorously, $0.8 \mathrm{~mL}$ of $8.5 \times 10^{-4} \mathrm{M}$ trisodium citrate $\left(\mathrm{Na}_{3} \mathrm{C}_{6} \mathrm{H}_{5} \mathrm{O}_{7}\right)$ was added all at once while heating and stirring for $30 \mathrm{~min}$. The solution then turned successively from light yellow to deep gray, looking finally dark red after $\sim 10 \mathrm{~min}$. The corresponding UV-visible absorbance spectrum of the resulting water solution of AuNPs was therefore obtained to monitor the position of the absorption peak. In order to graft AuNPs on the silicon Si(100) substrate, wafers of ultrasonically cleaned n-doped silicon (1 $\times 1 \mathrm{~cm}^{2}$, Siltronix) were silanised in an absolute methanol solution through 3-aminopropyltriethoxysilane (APTES, $\mathrm{H}_{2} \mathrm{~N}\left(\mathrm{CH}_{2}\right)_{3} \mathrm{Si}\left(\mathrm{OC}_{2} \mathrm{H}_{5}\right)_{3}, 10 \%$ vol.) over $90 \mathrm{~min}$. Afterwards, they were dipped in the AuNPs colloidal solution over $24 \mathrm{~h}$ in order to ensure a large surface density of AuNPs $N s \sim 8 \times 10^{10} / \mathrm{cm}^{2}$, corresponding to a filling factor $f \sim 15 \%$ as deduced by AFM measurements [40]. After grafting, AuNPs were functionalised during $18 \mathrm{~h}$ with a $10^{-3} \mathrm{M}$ thiophenol $\left(\mathrm{C}_{6} \mathrm{H}_{5} \mathrm{SH}\right)$ solution dissolved in dichloromethane $\left(\mathrm{CH}_{2} \mathrm{Cl}_{2}\right)$. We 
finally obtained a monolayer of AuNPs grafted to the APTES-Si surface and covered with thiophenol.

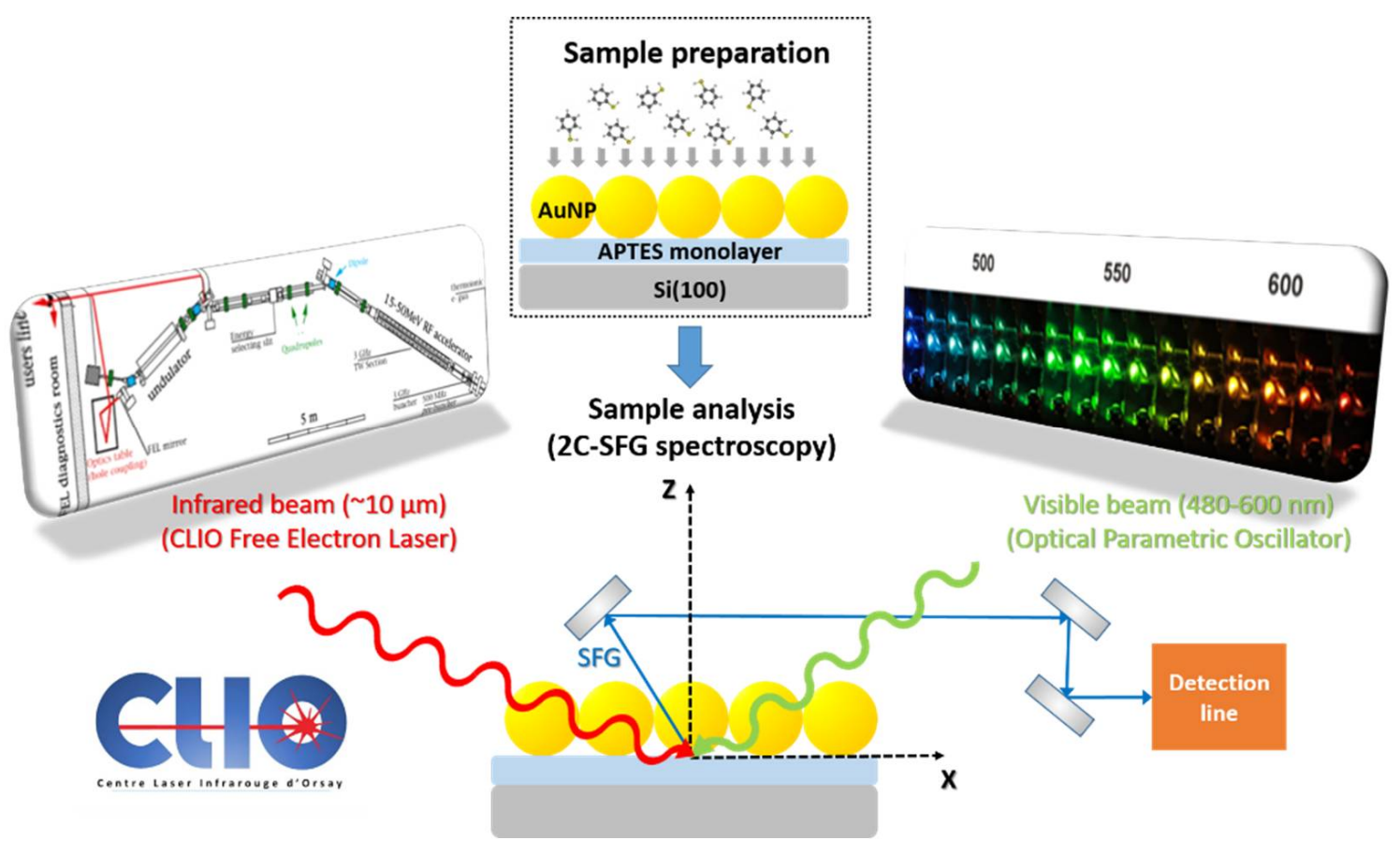

Figure 1. Sketch of the sample preparation and of the 2C-SFG experimental configuration used in counterpropagating geometry. See text for details.

\subsection{AFM Microscopy}

In order to analyse the surface density Ns of AuNPs grafted on the sample, an AFM imaging (Digital Instrument, DI3100, USA) was used in tapping mode. The working frequency of the silicon tip (curvature radius at the apex $\sim 10 \mathrm{~nm}$ ) was $130 \mathrm{KHz}$. This allowed us to count the AuNPs and measure their diameter $(17 \pm 2 \mathrm{~nm})$ equal to their height with respect to the $\mathrm{Si}(100)$ surface, even if it did not have a good lateral resolution to image isolated AuNPs.

\subsection{UV-Vis Spectroscopy}

Absorption (solution) and reflection (sample) spectroscopy measurements were performed through a Cary 5 spectrophotometer (Varian). On the silicon substrate, the spectra were recorded with an incidence angle of $10^{\circ}$. In order to detangle the AuNPs signature from the silicon contribution, the bare $\operatorname{Si}(100)$ reflectivity $R_{0}$ was first measured, prior to the sample reflectivity $R$ in the same conditions. Within this differential measurement procedure, it was therefore possible to access the sample reflectance $-\log \left(R / R_{0}\right)$ with a reproducibility ensured within $1 \times 10^{-3}$ A.U. (Absorbance Units).

\subsection{C-SFG Spectroscopy with the CLIO Free Electron Laser Facility}

A Sum-Frequency Generation (SFG) tabletop setup based on a Nd:YLF laser source at $1047 \mathrm{~nm}$ wavelength (repetition rate for macropulses: $25 \mathrm{~Hz}$; for micropulses: $62.5 \mathrm{MHz}$ ) was coupled to the CLIO Free Electron Laser (FEL) Facility with the same temporal features [37]. The CLIO FEL was used to take profit of its intense and pulsed IR laser beam ( $\sim 1$ ps pulse duration, $\sim 10 \mu \mathrm{J}$ energy per pulse from which $\sim 2 \mu \mathrm{J}$ were used in order to avoid sample damage, $\sim 5 \mathrm{~cm}^{-1}$ spectral resolution) to probe the vibration fingerprint of thiophenol molecules (ring deformations) in the $\sim 10 \mu \mathrm{m}$ spectral range $\left(975-1100 \mathrm{~cm}^{-1}\right.$ ). The incident visible laser beam $\left(\sim 5 \mathrm{ps}, 4 \mu \mathrm{J}\right.$ per pulse, $\sim 6 \mathrm{~cm}^{-1}$ spectral resolution) was tuned from $480 \mathrm{~nm}$ to $600 \mathrm{~nm}$ by $\sim 20 \mathrm{~nm}$ steps, resulting in 8 SFG spectra corresponding 
to 8 different incidence visible wavelengths. The tunable visible beam was obtained from a visible Optical Parametric Oscillator (OPO) based on the pumping of a rotating BBO (Barium Borate) nonlinear crystal inside an optical cavity by the 3rd harmonic (UV wavelength $=349 \mathrm{~nm}$ ) of the Nd:YLF laser source obtained after: first, the frequency-doubling (2nd harmonic visible wavelength $=523.5 \mathrm{~nm}$ ) through a BBO (Barium Borate) nonlinear crystal: second, the mixing of the remaining IR part with the resulting 2 nd harmonic in a LBO (Lithium Borate) nonlinear crystal. The IR and visible incident laser beams were mixed at the same point of the probed interface ( $2 \mathrm{~mm}$ laser beam diameter $)$ during the experiment. It is worth noting that the SFG experiments were performed in a counterpropagative geometry in order to well discriminate, spatially, the SFG beam from the reflected visible beam (by the silicon surface sample) going through a monochromator for spectral filtering. This original and unusual SFG spectroscopy configuration, aimed at avoiding light saturation of the photomultiplier located at the monochromator output, was derived from previous publications [29,33,41-44], allowing us to fix the detection direction for collecting SFG photons, whatever the IR or visible wavelengths. We improved the detection efficiency thanks to the use of rotating mirrors placed in the SFG path between the sample and the monochromator. Indeed, by considering the energy $\left(h \omega_{\mathrm{SFG}}=\mathrm{h} \omega_{\mathrm{Vis}}\right.$ $\left.+\mathbf{h} \omega_{I R}\right)$ and momentum ( $\mathbf{k}_{\mathrm{SFG}}=\mathbf{k}_{\mathrm{Vis}}+\mathbf{k}_{\mathrm{IR}}$ : valid for the parallel component only, i.e., in $\mathrm{x}$-direction, see Figure 1) conservation rules in SFG spectroscopy at $\sim 10 \mu \mathrm{m}$, whatever the chosen visible beam color, it was possible to well separate spatially the SFG beam from the latter. To meet these conditions, the IR and visible incidence angles of the laser beams were $65^{\circ}$ and $-55^{\circ}$ with respect to the sample surface normal (z-direction) as sketched in Figure 1. All beams were p-polarised. SFG data were normalised by the IR and visible powers in order to compensate for eventual laser fluctuations. All data are also corrected by the optical responses of photomultiplier and monochromator gratings which evolved from blue to orange-red.

\section{Results}

\subsection{AFM Characterisation}

Figure 2 displays the typical AFM image of our Si(100)/AuNPs/thiophenol surface. In order to deduce the AuNPs surface density $N s \sim 8 \times 10^{10} / \mathrm{cm}^{2}$, several scans were performed for different scales: $2 \times 2 \mu \mathrm{m}^{2}$ and $500 \times 500 \mathrm{~nm}^{2}$, allowing us to identify and count each individual nanoparticle (17 $\pm 2 \mathrm{~nm}$ diameter) by probing and averaging on at least five areas of the sample surface. The results obtained for the sample structural characterisation were consistent with the specific preparation (dipping of the grafted covered Si for $24 \mathrm{~h}$ in the AuNPs solution [38-40]) chosen to lead to AuNPs that were aggregated in close contact. This drastically modified the UV-Vis optical fingerprint of the sample with respect to the optical response in solution as shown thereafter.
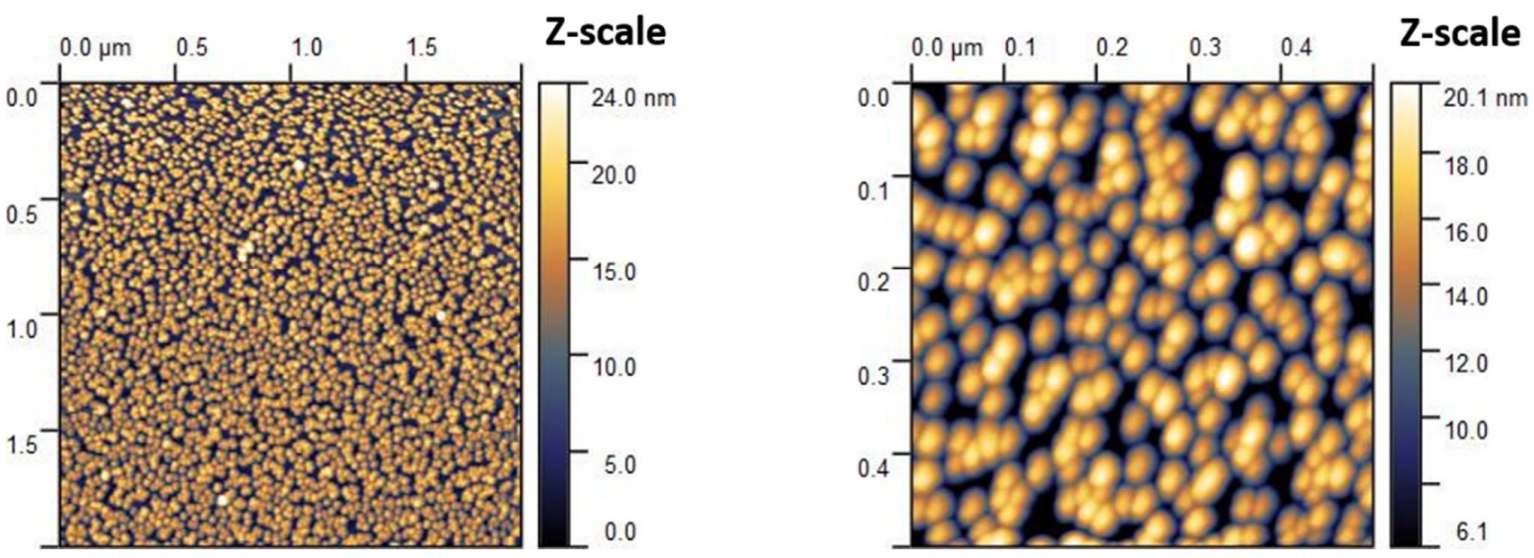

Figure 2. AFM image of the Silicon/APTES/AuNPs/Thiophenol interface. Left: $2 \times 2 \mu \mathrm{m}^{2}$ scale. Right: $500 \times 500 \mathrm{~nm}^{2}$ scale. Z-scale intensity: height. 


\subsection{UV-Visible Measurements}

Figure 3a displays the UV-Vis absorption spectrum of the AuNPs solution while Figure $3 \mathrm{~b}$ displays the UV-Vis reflectance spectrum of the $\mathrm{Si}(100) /$ AuNPs/thiophenol sample. The AuNPs absorbance spectrum shows a peak at $520 \mathrm{~nm}$, corresponding to their Localised Surface Plasmon Resonance (LSPR). When AuNPs were grafted on the $\mathrm{Si}(100)$ surface, the LSPR is dip-shaped and slightly red-shifted to $525 \mathrm{~nm}$. A second strong dip-shaped band appeared at $650 \mathrm{~nm}$ in the optical response. The latter one is directly related to AuNPs aggregation occurring during the deposition process on the surface (see Figure 2). This is a well-known phenomenon, demonstrated both theoretically and experimentally for metal nanoparticles in colloidal solutions or deposited with high density on substrates. In fact, this band results from coupling processes of surface plasmons between nanoparticles in close contact (interparticles coupling) [45]. To observe this kind of collective plasmon modes, the AuNPs were physically connected through aggregation, leading to a dipolar coupling between neighboring plasmonic oscillators, which was the case when the spacing between them was smaller than ca. $30 \mathrm{~nm}$. [46]. For instance, this kind of plasmonic collective coupling is used in biosensing to obtain a fine monitoring of the plasmon resonance position by playing at will for instance with gold nanorods size (aspect ratio) and spacing [47,48]. Besides, the two negative spectral shapes observed in Figure $3 \mathrm{~b}$ on the silicon surface are in relation with the reflectivity measurement mode, because the reflectivity of the silicon was strongly modulated by the presence of a AuNPs layer. Qualitatively, the more gold on the surface, the higher the reflectivity $R$, compared to the reference $R_{0}$. Therefore, the presence of AuNPs induced negative values for the reflectance as observe in Figure 3b. This optical phenomenon is explained in previous works $[40,49]$ based on the calculation of the dielectric constant of the AuNPs layer in the framework of the effective medium theory (Maxwell-Garnett and Bruggeman formalisms of low and high density composite layer, respectively).
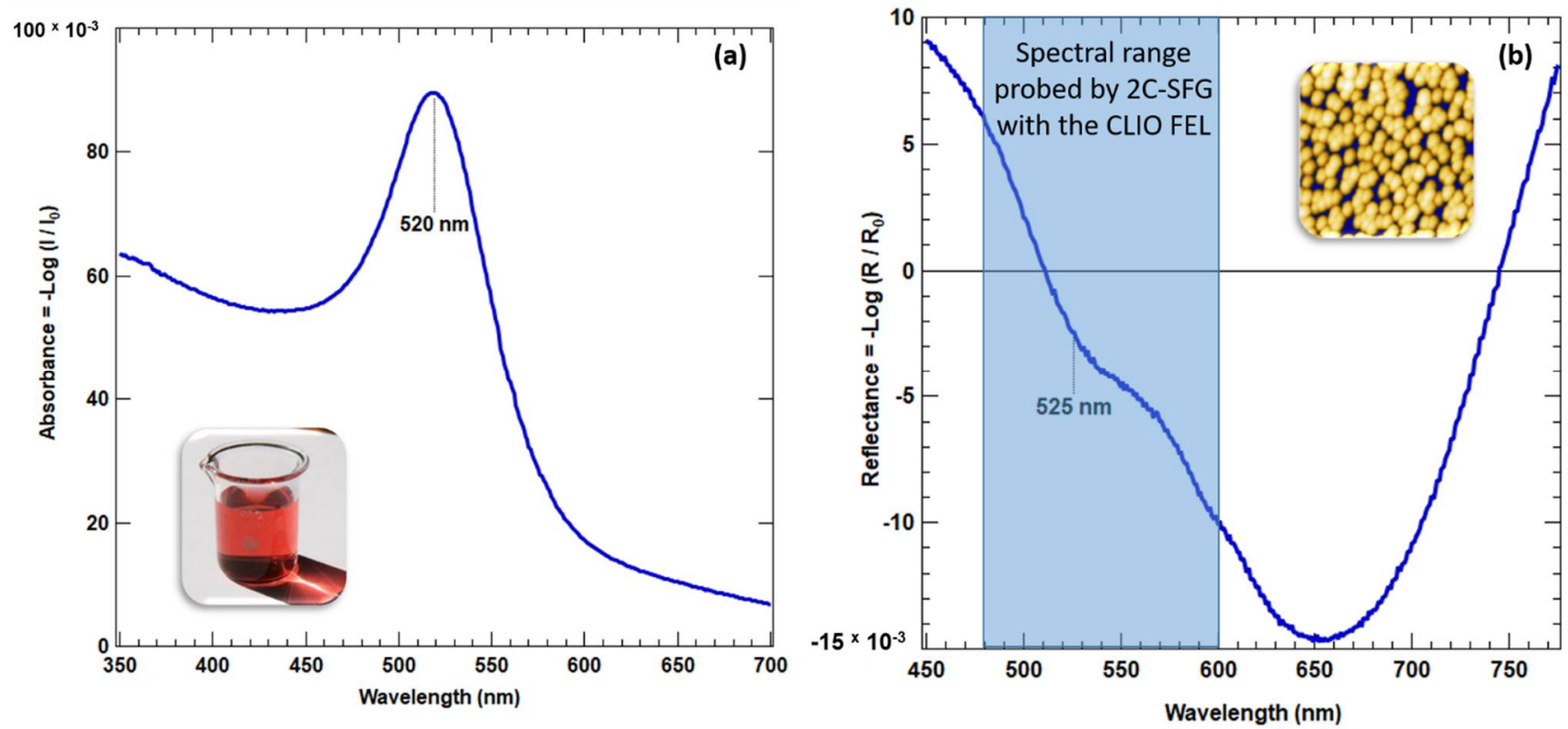

Figure 3. UV-Vis absorption (a) and reflectance (b) spectra of AuNPs colloidal solution (insert) and $\mathrm{Si}(100) /$ AuNPs/thiophenol sample (insert), respectively. The peak or valley shape at 520 and $525 \mathrm{~nm}$ wavelengths correspond to the LSPR of the AuNPs.

\subsection{C-SFG Measurements}

2C-SFG measurements of our sample made of $17 \mathrm{~nm}$ diameter AuNPs grafted on silicon and functionalised by thiophenol are recorded in counter-propagating geometry and depicted in Figure 4 for 8 different visible laser wavelengths from blue to orange-red 
as pointed out on the right of the panel. For each visible wavelength, three vibration modes of thiophenol molecules were observed at 1000, 1023 and $1071 \mathrm{~cm}^{-1}$, respectively.

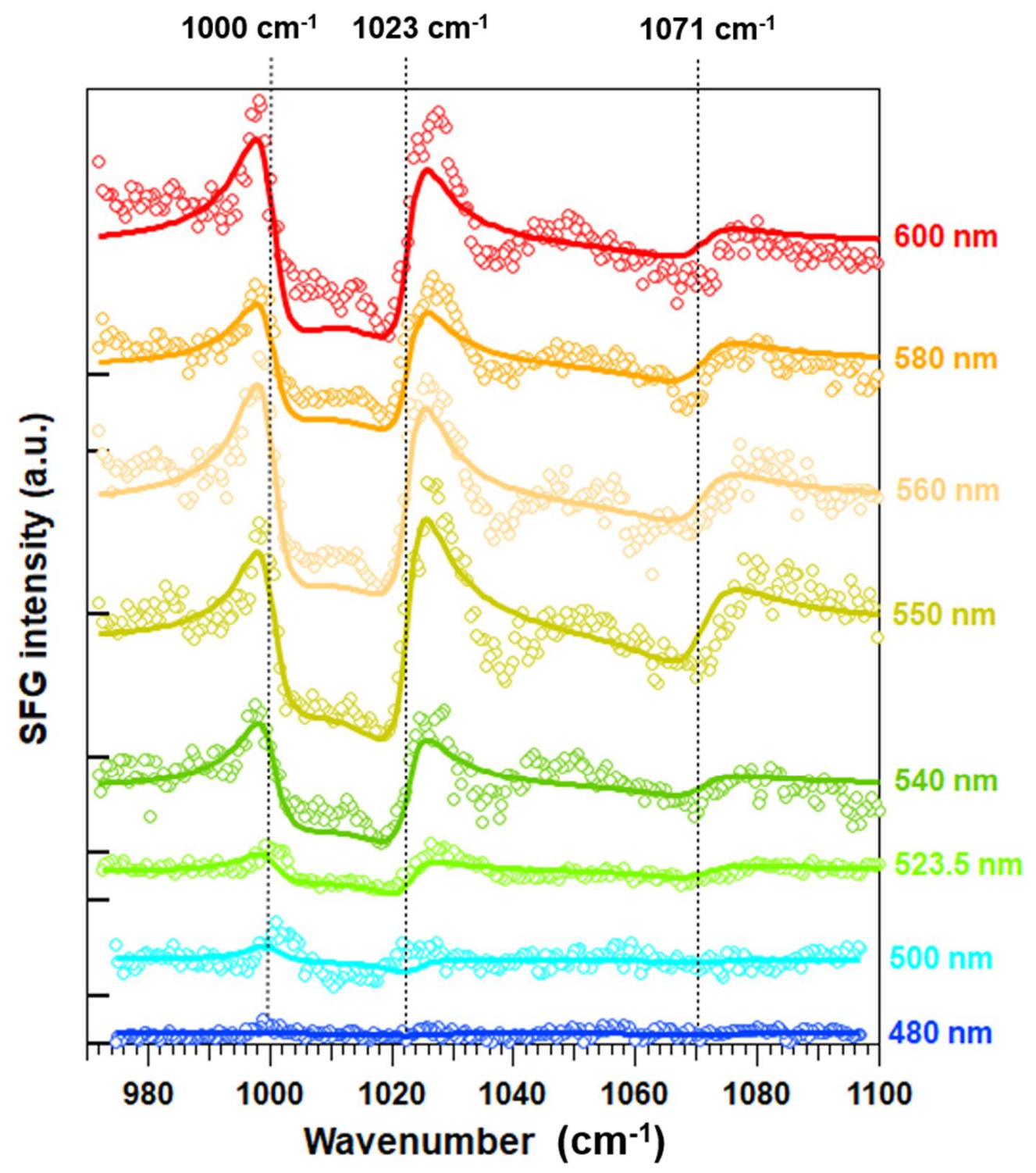

Figure 4. Experimental SFG spectra (open circles) obtained with the CLIO FEL for the $\mathrm{Si}(100) /$ AuNPs/thiophenol sample at 8 different visible beam wavelengths of the incident laser beam, ranging from blue to orange-red as noted on the right of the panel. On the left axis, the 0 of each curve is sketched by a short black line. While the SFG spectra are all shifted to ease view, they are all drawn within the same intensity scale to have a direct qualitative comparison. The three main vibration modes tagged at $1000 \mathrm{~cm}^{-1}, 1023 \mathrm{~cm}^{-1}$ and $1071 \mathrm{~cm}^{-1}$ correspond to specific phenyl ring deformations of the thiophenol molecules as established in the literature [31,39]. Colored lines are fitted to the SFG experimental data, as detailed and explained in the text. The SFG spectrum at $480 \mathrm{~nm}$ visible wavelength will act as the reference to calculate the plasmonic coupling effect and the amplification factor of the thiophenol vibrational fingerprint.

In 2C-SFG spectroscopy, a second order nonlinear optical process, it is counter intuitive to observe a signal from functionalised spherical objects because they are centrosymmetric media. In other words, there is a symmetry inversion center of the electronic properties (charge distribution) for an observer placed at the center of the gold spheres. A quick look on the sample structure given in Figure 1 shows us the reason for this apparent contradiction. The grafting of the AuNPs induced a symmetry breaking of the electronic 
properties in the z-direction where the ppp polarisation scheme of the three beams (SFG, Visible, IR) was the most efficient for 2C-SFG spectroscopy. Moreover, the AuNPs were mostly functionalised by thiophenol molecules on their upper surface, while they were not on their lower surface, grafted on silicon through APTES molecules. While AuNPs are not, in principle, the most efficient objects for second order nonlinear optics, the current work shows that it is again possible to detect such molecular species on small metal nanospheres and, more interestingly, to evidence the positive role of the plasmonic coupling effect in improving the detection efficiency of 2C-SFG spectroscopy. Besides, it has been shown that the SFG vibration mode amplitude of thiophenol molecules increased linearly with Ns (AuNPs surface density): the more the AuNPs were aggregated, the higher the SFG process efficiency [40]. In fact, the first striking feature observed in Figure 4 is the effect of the selected visible laser beam wavelengths on the SFG signal of the thiophenol vibrational fingerprint. It is clear that the three vibration modes were difficult to observe in the blue spectral range for a common intensity scale but they became more and more apparent with the increasing visible wavelength to the orange-red. The second striking feature is the Fanolike shape of these three vibration modes, i.e., different from valleys or peaks as observed in IR and Raman spectroscopy. This is specific to 2C-SFG spectroscopy as a sensitive tool of the interface constituting the sample. As a result, the two predominant contributions originate from thiophenol molecules and the AuNPs surface where they were adsorbed. In a general way, the SFG intensity writes: $\mathrm{I}\left(\omega_{\mathrm{SFG}}\right)=\frac{8 \pi^{3} \omega_{\mathrm{SFG}}^{2} \sec ^{2} \theta_{\mathrm{SFG}}}{c^{3} n_{1}\left(\omega_{\mathrm{SFG}}\right) n_{1}\left(\omega_{\mathrm{IR}}\right) n_{1}\left(\omega_{\mathrm{vis}}\right)}\left|\chi_{\mathrm{eff}}^{(2)}\right|^{2} \mathrm{I}_{\mathrm{IR}} \mathrm{I}_{\mathrm{vis}}$ with $\mathrm{I}_{\mathrm{IR}}$ and $\mathrm{I}_{\mathrm{Vis}}$ the intensities of the incident IR and visible laser beams, respectively. $\omega_{I R}, \omega_{\text {vis }}$ and $\omega_{\mathrm{SFG}}$ are the IR, visible and sum frequencies (energies) of the three beams involved in the nonlinear second order process. The incoming angles of incidence of the IR and visible beams are $\theta_{\mathrm{IR}}$ and $\theta_{\mathrm{vis}}$ while $\theta_{\mathrm{SFG}}$ is the outgoing angle of incidence of the SFG beam travelling to the monochromator as depicted in Figure 1. From the sample point of view, $\mathrm{n}_{1}(\omega)$ is the refractive index of the upper medium (ambient air) at frequency $\omega$ and $\chi_{\text {eff }}^{(2)}$ the effective nonlinear second order susceptibility of the probed interface. The latter includes Fresnel factors (sample reflectivity) and microscopic local-field corrections to the (thiophenol) molecular nonlinear 2 nd order susceptibilities $\chi_{\mathrm{ijk}}^{(2)}$. In these conditions, we model $\chi_{\text {eff }}^{(2)}=\left\|C_{N R}\right\| \mathrm{e}^{\mathrm{i} \phi_{\mathrm{NR}}}+\sum_{\mathrm{n}} \frac{\left\|\mathrm{a}_{\mathrm{n}}\right\| \mathrm{e}^{\mathrm{i} \varphi_{\mathrm{n}}}}{\left(\omega_{\mathrm{IR}}-\omega_{\mathrm{n}}+\mathrm{i} \Gamma_{\mathrm{n}}\right)} \quad$ leading to the colored lines in good agreement with the experimental data points depicted in Figure 4. The first complex term (amplitude $\mathrm{C}_{\mathrm{NR}}$ and phase shift $\Phi_{\mathrm{NR}}$ ) of the above equality corresponds to the substrate (AuNPs) contribution, which is non-resonant (NR) with IR energy. The second complex term is the sum of the three Lorentzian oscillators of the thiophenol molecules $(n=3)$ observed in the 975-1100 $\mathrm{cm}^{-1}$ spectral range, with $a_{n}, \varphi_{n}, \omega_{n}$ and $\Gamma_{n}$ the amplitude, phase, frequency and damping constant of each vibration mode, respectively. It is worth noting that the complex value $a_{n}$ is a combination of the IR transition dipole moments and Raman polarisability tensors of the molecule. The physical meaning and role of those fundamental parameters are exhaustively detailed in recent publications covering both a classical point of view [27] and advanced quantum formalism [50]. For the fitting of the set of SFG data, we used, for all spectra fitting procedure, the known fixed fit parameters for the SFG spectrum at $523.5 \mathrm{~nm}$. Indeed, for similar samples, it has been established previously [39] that the thiophenol frequencies are $\omega_{\mathrm{n}}\left(\mathrm{cm}^{-1}\right)=1000,1023,1071$, while the damping constants are set to $\Gamma_{\mathrm{n}}\left(\mathrm{cm}^{-1}\right)=3.5,3.5,5$ and $\varphi_{\mathrm{n}}\left({ }^{\circ}\right)=0$ for $n=1,2,3$, respectively. The results of the fitting procedure are summarised in Table 1 . The observed variation of SFG data with visible wavelength is thus mainly related to a significant increase of the amplitude absolute value $a_{n}$ of the three thiophenol vibration modes when moving from blue to orange-red. A similar behavior was observed for AuNPs amplitude $C_{N R}$ but on a smaller relative scale. We define the intensity ratio $I_{n}^{\text {ratio }}=\left\|a_{n} / C_{N R}\right\|^{2}$ as calculated in Table 1 for the three thiophenol vibration modes and for each visible wavelength. $I_{n}^{\text {ratio }}$ increased by a factor of $\sim 100$ from blue $(480 \mathrm{~nm})$ to green-yellow $(540-560 \mathrm{~nm})$, and remained almost stable in the orange-red spectral range. In the current work, we have chosen to consider that the reference SFG measurement was the one at $480 \mathrm{~nm}$ (blue) in 
order to calculate the plasmonic coupling effect on the amplification factor of the SFG vibrational molecular fingerprint. Indeed, no plasmonic effect and no significant s-d interband electronic transition competed with the SFG process efficiency when looking at the UV-Visible reflectance curve in the $480 \mathrm{~nm}$ spectral range depicted in Figure 3b. Comparative reference SFG measurements between an identical sample with respect to a flat gold surface covered by thiophenol performed in a previous work [38] demonstrated that the amplification factor by the AuNPs was around 21 (for thiophenol SFG intensity) at a fixed $532 \mathrm{~nm}$ visible wavelength. A comparison with thiophenol adsorbed on silicon or glass is not relevant from the chemical and optical point of view because: (i) thiophenol does not adsorb on silicon or glass; (ii) only a comparison between a flat vs. a curved gold surface takes account of the plasmonic coupling effect for similar chemical conditions of molecular adsorption. In other words, we observed, in the best conditions of our SFG measurements of Figure 4, an amplification factor of two orders of magnitude for the SFG intensity of the thiophenol vibration modes as a function of the laser color impinging the AuNPs with respect to the reference SFG spectrum with $480 \mathrm{~nm}$ visible wavelength. Besides, a $\sim 65^{\circ}$ phase shift $\Phi_{\mathrm{NR}}$ parameter evolving from blue to orange-red in the fitting procedure allowed us to adjust the interference pattern evolution of the thiophenol vibration modes modulated by gold electronic properties, as explained hereafter.

Table 1. Fit parameters for the 2C-SFG spectra of thiophenol adsorbed on AuNPs as obtained from Figure 4. For $\varphi_{n}, \omega_{n}$ and $\Gamma_{n}$, we refer to fixed parameters for the three thiophenol vibration modes as established in [39]. See text for details.

\begin{tabular}{|c|c|c|c|c|c|c|c|c|c|}
\hline & & \multicolumn{8}{|c|}{ Visible Incidence Wavelength (nm) } \\
\hline & & 480 & 500 & 523.5 & 540 & 550 & 560 & 580 & 600 \\
\hline \multirow{3}{*}{ AuNPs } & $\mathrm{C}_{\mathrm{NR}}$ & 1.4310 & 2.6538 & 2.3970 & 3.5530 & 4.8961 & 4.63128 & 4.0848 & 4.9943 \\
\hline & $\Phi_{\mathrm{NR}}\left(^{\circ}\right)$ & 69.8753 & 59.4616 & 28.0546 & 12.6793 & 11.6879 & 5.5153 & 7.5741 & 4.6585 \\
\hline & $\begin{array}{c}\mathrm{a}_{1} \text { (a.u.) } \\
\varphi_{1}\left({ }^{\circ}\right)\end{array}$ & -0.2376 & -1.8922 & -3.6383 & -9.5138 & $\begin{array}{l}-10.931 \\
0\end{array}$ & -14.104 & -9.3308 & -12.517 \\
\hline \multirow[t]{4}{*}{ Vibr 1} & $\begin{array}{c}\omega_{1} \\
\left(\mathrm{~cm}^{-1}\right)\end{array}$ & & & & & 1000 & & & \\
\hline & $\Gamma_{1}\left(\mathrm{~cm}^{-1}\right)$ & & & & & 3.5 & & & \\
\hline & $I_{1}^{\text {ratio }}$ & 0.02757 & 0.5084 & 2.3039 & 7.17 & 4.9845 & 9.2743 & 5.2179 & 6.2813 \\
\hline & $\begin{array}{c}\mathrm{a}_{2} \text { (a.u.) } \\
\varphi_{2}\left({ }^{\circ}\right)\end{array}$ & 0.5194 & 1.6344 & 5.2915 & 10.8236 & $\begin{array}{l}16.4952 \\
0\end{array}$ & 14.3315 & 10.3851 & 11.763 \\
\hline \multirow[t]{4}{*}{ Vibr 2} & $\begin{array}{c}\omega_{2} \\
\left(\mathrm{~cm}^{-1}\right)\end{array}$ & & & & & 1023 & & & \\
\hline & $\Gamma_{2}\left(\mathrm{~cm}^{-1}\right)$ & & & & & 3.5 & & & \\
\hline & $I_{2}^{\text {ratio }}$ & 0.1317 & 0.3793 & 4.8733 & 9.2801 & 11.3505 & 9.5759 & 6.4637 & 5.5474 \\
\hline & $\mathrm{a}_{3}$ (a.u.) & 0.2721 & 0.7215 & 2.6407 & 3 & 7.8051 & 5.2497 & 5 & 3 \\
\hline \multirow{4}{*}{$\begin{array}{l}\text { Vibr } 3 \text { (Mode } 4 \\
\quad \text { in }[31,39])\end{array}$} & $\varphi_{3}\left({ }^{\circ}\right)$ & & & & & 0 & & & \\
\hline & $\begin{array}{c}\omega_{3} \\
\left(\mathrm{~cm}^{-1}\right)\end{array}$ & & & & & 1071 & & & \\
\hline & $\Gamma_{3}\left(\mathrm{~cm}^{-1}\right)$ & & & & & 5 & & & \\
\hline & $I_{3}^{\text {ratio }}$ & 0.0361 & 0.0739 & 1.2137 & 0.7129 & 2.5413 & 1.2849 & 1.4983 & 0.3608 \\
\hline
\end{tabular}

\section{Discussion}

\subsection{Phase Shift Parameter: Gold s-d Interband Electronic Transitions}

The evolving spectral shapes take their origin in the interference phenomenon between the SFG signals of the molecular adsorbate (thiophenol) and the substrate (AuNPs). This is well explained in the literature on gold surfaces and related to its s-d interband electronic transitions [51]. On a flat gold surface covered by a monolayer of dodecanethiol (DDT), a similar decreasing phase shift of $\sim 65^{\circ} \Phi_{\mathrm{NR}}$ was observed between 480 and $600 \mathrm{~nm}$, inducing a shape reversal of the vibration modes when moving from a blue visible incidence beam to a red one. This is related to the different electronic contributions of the gold surface and bulk properties (free and bound electrons of the electronic density of states of gold, see 
Figure 5, right) as exhaustively developed elsewhere [52,53], giving access to the dispersion of the absolute phase and the relative amplitude of its $\chi_{\mathrm{eff}}^{(2)}$ in the visible spectral range. It allows us to model quantitatively SFG spectra of molecules adsorbed on metal surfaces. Our SFG measurements are performed and analysed in the same theoretical framework, leading to consistent interpretations and conclusions.

\section{Gold Plasmonic contribution}

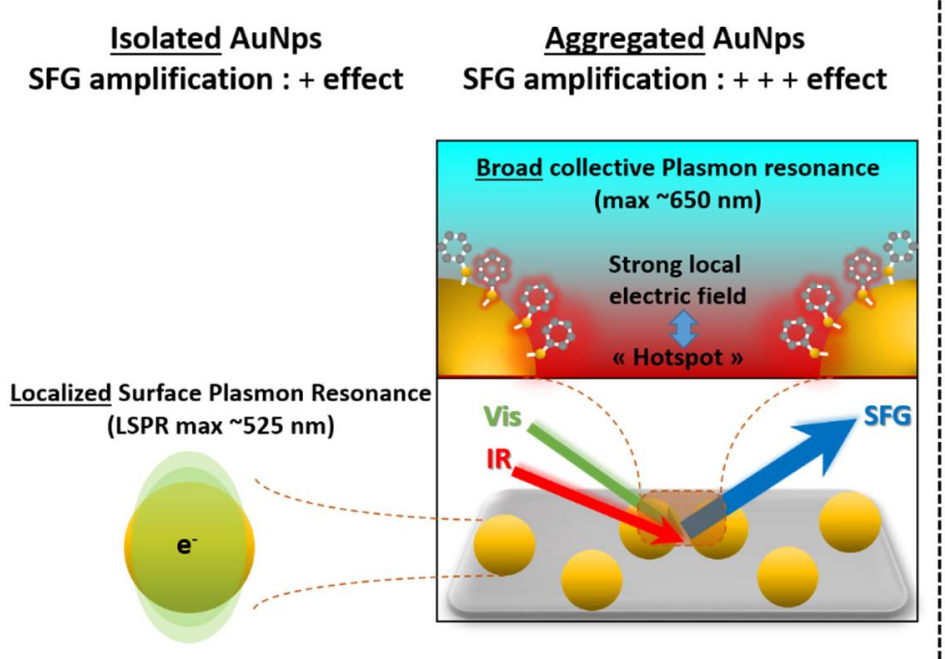

\section{Gold Bulk contribution}

AuNps Bulk

SFG amplification : - effect

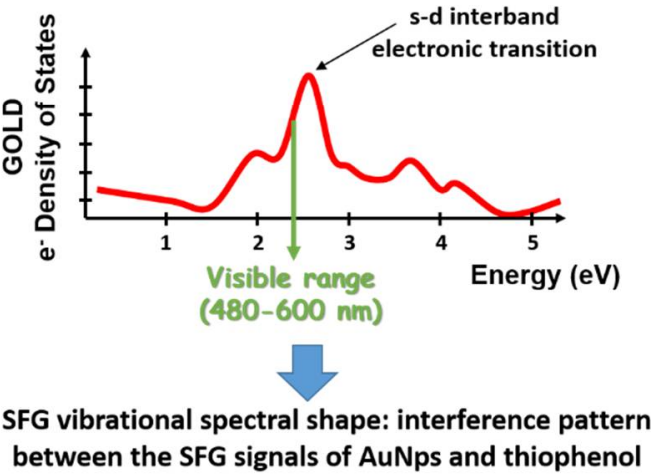

Figure 5. Sketch of the physical processes competing positively $(+)$ or negatively $(-)$ in the amplification of the thiophenol vibrational fingerprint for 2C-SFG spectroscopy coupled to plasmonics based on small $17 \mathrm{~nm}$ diameter AuNPs grafted on a silicon substrate. The LSPR and collective plasmon modes of AuNPs contributed favourably (left), especially as the number of hotspots increased on the sample. The surface and bound electrons of the gold bulk of AuNPs contributed unfavourably (right) due to the high electronic density of states in the probed visible (480-600 nm) spectral range. The latter physical process also explains the interference pattern between the SFG signals of the thiophenol molecules and the AuNPs constituting the interface. See text and references [51-53] for details on the influence of gold bulk electronic properties.

\subsection{Co-vs. Counter-Propagating Geometry: SFG Spectra Shape Reversal}

A notable difference is observed for the SFG spectrum at $523.5 \mathrm{~nm}$ wavelength with respect to reference [39], where a similar measurement was recorded, but in co-propagating classical geometrical configuration for the three beams involved in the SFG process: the shape of the three vibration modes displayed a different interference pattern. In practice, $\Phi_{\mathrm{NR}}=\sim 140^{\circ}$ in [39] while $\Phi_{\mathrm{NR}}=\sim 30^{\circ}$ in our current work in counter-propagating geometry. The only difference between these two works lies in the setup geometry; we can explain the phase shift difference $\geq \pi / 2$ by an inversion symmetry of the visible beam incidence angle as depicted in Figure 1. The sign of the x-components of the probed $\chi_{\mathrm{eff}}^{(2)}$ is inverted with respect to this previous work in such a way that the SFG vibrational spectral shapes are, therefore, reversed.

\subsection{Amplification of Thiophenol Vibration Modes: Collective Plasmon Modes Coupling}

In order to interpret the striking amplification factor, increasing as a function of laser color, we have to consider the optical properties of our $17 \mathrm{~nm}$ diameter AuNPs where thiophenol molecules are grafted, that are characterised by plasmon resonances (LSPR for isolated particles and collective modes for aggregated particles) and s-d interband electronic transitions (see Figure 5). In our case, due to the strong aggregation, the collective plasmon modes already coupled very efficiently to the SFG process (thiophenol vibration modes intensity) showing a continuous significant enhancement of the SFG intensity when 
the color was tuned from blue to orange-red. Nevertheless, its efficiency became slightly damped/stabilised beyond $560 \mathrm{~nm}$, as observed in Figure 4 and Table 1, because of the more prominent role played by the surface and bound electrons of bulk gold in this spectral range. These two competing physical processes are summarised in Figure 5. The plasmonic coupling effect is similar to those observed and modeled in previous works on alkane thiol chains $[1,17,23,24]$ but with a greater efficiency in the present case because we were able to probe selectively better conditions with the tunable visible beam, which was not the case in the aforementioned works. Indeed, we observed here a vibrational SFG intensity amplification of two orders of magnitude for thiophenol molecules, whereas it was only of around one order of magnitude for alkanethiol chains (DDT). This result can be explained by the combination of the following factors. Firstly, by considering our AFM images in Figure 2 and UV-Visible spectrum in Figure $3 b$, we had the substrate surface completely filled with AuNPs in tight contact, forming numerous aggregates. This constituted the best conditions to obtain numerous hotspots between AuNPs (Figure 5), with strong local electric fields where thiophenol molecules were bathing. These numerous hotspots added to the LSPR enhancement process alone [1] due to the resulting existence of collective plasmon modes as explained in Section 3.2. In our case, it was highlighted by the UV-Vis intensity continuous increase observed in Figure $3 b$, beyond the local LSPR maximum $(525 \mathrm{~nm})$ from blue to red colors in our SFG framework. Secondly, thiophenol molecules are of shorter length than alkanethiol molecules. The three vibration modes observed in Figure 4 underwent a stronger influence from the local electric field than the methyl end-groups $\left(\mathrm{CH}_{3}\right)$ of DDT. Thirdly, the thiophenol phenyl rings involved in the SFG process have a rich $\pi$-electronic structure with respect to DDT. All these factors come into play to explain our observations, as well as the fact that phenyl rings present well-known intense Raman active modes in the $\sim 1000 \mathrm{~cm}^{-1}$ spectral range [30,31]. Since vibro-electronic 2CSFG spectroscopy depends on IR transition dipole moments and Raman polarisability of molecules, further investigation will necessitate different polarisation combinations of the three beams involved in the nonlinear process in order to give access to the different components of the molecular nonlinear second order susceptibilities $\chi_{\mathrm{ijk}}^{(2)}$.

To go further, considering the AuNPs' size effect on these two physical values would reveal the following. For the aggregation effects $(650 \mathrm{~nm})$, it would only shift the position and broaden the maximum absorption in the optical range of Figure 3b. From the optical point of view, it corresponds to tune the visible wavelength in the SFG process to find the best amplification factor for the plasmonic coupling effect. It could be very interesting for dedicated applications requiring a fine monitoring of the plasmon resonance position in the visible range as encountered for gold nanorods where it is possible to play with their aspect ratio and their spacing, such as in biosensing $[47,48]$, as already mentioned in Section 3.2. For the s-d interband electronic transition, it will increase the non-resonant contribution from blue to red ( $\mathrm{C}_{\mathrm{NR}}$ parameter in Table 1$)$ of the gold SFG signal because it is a bulk effect, with no change in the position of the s-d interband electronic transition as illustrated in Figure 5. It is worth noting that an increasing value of $C_{N R}$ (with an increasing AuNPs size) will compete negatively with the plasmonic amplification factor.

\subsection{Technical Prospects for 2C-SFG Spectroscopy}

In order to analyse more precisely the role of AuNPs LSPR and hotspots, it will be necessary to gain a wider visible spectral range in direction of red colors. Besides, it should be noted that recording data beyond $10 \mu \mathrm{m}$ in 2C-SFG spectroscopy will require better detection (spatial and spectral filtering) conditions, because the SFG beam is emitted in a direction almost colinear to the reflected visible beam with separation angles $<1^{\circ}$ in co-propagating geometry. It is consequently difficult to extract one SFG photon from the surrounding visible noise $10^{6}$ times more intense that the nonlinear processes. The counterpropagating geometry proposed here gives an original alternative to sweep this problem in the mid/far IR range but remains demanding from the experimental point of view. Besides, it is worth noting that just increasing the visible laser beam power to compensate for weak 
IR power in the $10 \mu \mathrm{m}$ spectral range is not adapted for such nanostructured samples, explaining the lack of results published in the scientific literature. Indeed, increasing the visible beam energy will quickly damage (heating and burning) the sample, the aromatic rings of thiophenol molecules first, then the AuNPs and the silicon substrate next. This is related to the fact that the required mean visible energy distributed for large laser beam scale ( $2 \mathrm{~mm}$ diameter for IR and Visible beams in our case) on the sample to generate a nonlinear process becomes very important. There is a power balance to find with the required IR energy threshold which enhances specifically the vibration modes of thiophenol molecules (and is Non-Resonant for gold), knowing that the two IR and visible beams have the same spot size at the same point of the probed interface. In these conditions, the local electric field between hotspots being amplified by a factor of $10^{3}-10^{6}$ is commonly observed in Raman spectroscopy at the molecular scale, so it is by far more interesting to use plasmonic amplification coupled to enough IR power to favour and enhance the SFG process to have a good spectral resolution. In other words, for nonlinear optics coupled to plasmonics, in order to have enough visible energy is a necessary condition but not sufficient for vibrational spectroscopy. A continuous effort in the development of table-top IR fs laser sources remains mandatory and challenging.

\section{Conclusions}

The CLIO Free Electron Laser has been revealed as a powerful and pioneering tool when coupled to SFG spectroscopy, giving a unique optical setup that led to numerous experimental firsts during 25 years of operation, thanks to its unique delivered power in the infrared spectral range, opening the door to nonlinear optical spectroscopy in the vibrational fingerprint as illustrated in the present work. We highlighted a plasmon-molecular coupling by 2C-SFG spectroscopy with an intensity amplification factor around two orders of magnitude from blue to green-yellow due to the presence of numerous hotspots between aggregates of small gold nanospheres functionalised by molecular thiophenol probes. Thanks to our work, if we combine the amplification factor obtained by comparing the SFG signal of thiophenol adsorbed on a flat gold surface with respect to thiophenol adsorbed on AuNPs ( 20), with the one calculated here ( 100), we tend to reach a global amplifcation factor of the SFG intensity of $10^{3}$. This must be put in perspective with the commonly and easily reached better Raman amplification factors $\left(10^{3}-10^{6}\right)$, knowing that: (i) our sample is made of small spherical objects, far from being the best conditions for 2C-SFG spectroscopy (centrosymmetry to be broken); (ii) conversely, the Raman spectroscopy efficiency become limited with decreasing size of nano-objects. Indeed, for Raman, smaller means greater light diffusion cross section, especially below $100 \mathrm{~nm}$, damping drastically the molecular sensitivity in an intrinsic Raman signal background from gold nanospheres. Our prominent and promising results show that nonlinear plasmonics constitutes an efficient probe of constantly increasing threshold sensitivity. The golden age of surface nonlinear optical spectroscopy is only at its beginning.

Author Contributions: Conceptualisation of the 2C-SFG setup coupled to the CLIO FEL, A.T., B.B. and C.H.; sample preparation, O.P. and C.H.; UV-Vis and AFM characterisation, O.P. and E.L.; 2C-SFG measurements, C.H. and B.B.; 2C-SFG data treatment and analysis, C.H.; writing-original draft preparation, C.H.; writing-review and editing, C.H., O.P., E.L., B.B. and A.T.; funding acquisition of the 2C-SFG setup, A.T. All authors have read and agreed to the published version of the manuscript.

Funding: This research was financially supported by the EU Horizon 2020 Programme (CALIPSOPlus under grant number 730872).

Data Availability Statement: The data presented in this study are available on reasonable request from the corresponding author.

Acknowledgments: The authors acknowledge Research Engineer C. Six, Assistant Engineer A. Gayral for their continuous technical assistance in the proper functioning of the 2C-SFG spectroscopy setup, Research Engineer J.-P. Berthet, Research Director J.-M. Ortega for their technical and scientific support for FEL operation. C. Humbert also acknowledges all the past and present scientific (F. Glotin, 
R. Prazeres) and technical (J. Vieira, G. Périlhous, F. Gobert, V. Fèries, B. Rieul and N. Jestin, Technical Head of the CLIO FEL) staff met at the CLIO Facility since year 1997 during his Master Internship until this day.

Conflicts of Interest: The authors declare no conflict of interest.

\section{References}

1. Dalstein, L.; Humbert, C.; Ben Haddada, M.; Boujday, S.; Barbillon, G.; Busson, B. The Prevailing Role of Hotspots in PlasmonEnhanced Sum-Frequency Generation Spectroscopy. J. Phys. Chem. Lett. 2019, 10, 7706-7711. [CrossRef]

2. Lis, D.; Caudano, Y.; Henry, M.; Demoustier-Champagne, S.; Ferain, E.; Cecchet, F. Selective Plasmonic Platforms Based on Nanopillars to Enhance Vibrational Sum-Frequency Generation Spectroscopy. Adv. Opt. Mater. 2013, 1, 244-255. [CrossRef]

3. Lis, D.; Cecchet, F. Localized surface plasmon resonances in nanostructures to enhance nonlinear vibrational spectroscopies: Towards an astonishing molecular sensitivity. Beilstein J. Nanotechnol. 2014, 5, 2275-2292. [CrossRef] [PubMed]

4. Noblet, T.; Dreesen, L.; Boujday, S.; Méthivier, C.; Busson, B.; Tadjeddine, A.; Humbert, C. Semiconductor quantum dots reveal dipolar coupling from exciton to ligand vibration. Commun. Chem. 2018, 1, 76. [CrossRef]

5. Noblet, T.; Boujday, S.; Méthivier, C.; Erard, M.; Hottechamps, J.; Busson, B.; Humbert, C. Two-Dimensional Layers of Colloidal CdTe Quantum Dots: Assembly, Optical Properties, and Vibroelectronic Coupling. J. Phys. Chem. C 2020, 124, 25873-25883. [CrossRef]

6. Sengupta, S.; Bromley, L.; Velarde, L. Aggregated States of Chalcogenorhodamine Dyes on Nanocrystalline Titania Revealed by Doubly Resonant Sum Frequency Spectroscopy. J. Phys. Chem. C 2017, 121, 3424-3436. [CrossRef]

7. Dreesen, L.; Sartenaer, Y.; Humbert, C.; Mani, A.A.; Lemaire, J.-J.; Méthivier, C.; Pradier, C.-M.; Thiry, P.A.; Peremans, A. Sum-frequency generation spectroscopy applied to model biosensors systems. Thin Solid Films 2004, 464-465, 373-378. [CrossRef]

8. Humbert, C.; Noblet, T.; Dalstein, L.; Busson, B.; Barbillon, G. Sum-Frequency Generation Spectroscopy of Plasmonic Nanomaterials: A Review. Materials 2019, 12, 836. [CrossRef] [PubMed]

9. Noblet, T.; Dreesen, L.; Tadjeddine, A.; Humbert, C. Spatial dependence of the dipolar interaction between quantum dots and organic molecules probed by two-color sum-frequency generation spectroscopy. Symmetry 2021, 13, 1636. [CrossRef]

10. Dreesen, L.; Humbert, C.; Sartenaer, Y.; Caudano, Y.; Volcke, C.; Mani, A.A.; Peremans, A.; Thiry, P.A.; Hanique, S.; Frère, J.-M. Electronic and molecular properties of an adsorbed protein monolayer probed by two-color sum-frequency generation spectroscopy. Langmuir 2004, 20, 7201-7207. [CrossRef]

11. Raab, M.; Becca, J.C.; Heo, J.; Lim, C.K.; Baev, A.; Jensen, L.; Prasad, P.N.; Velarde, L. Doubly resonant sum frequency spectroscopy of mixed photochromic isomers on surfaces reveals conformation-specific vibronic effects. J. Chem. Phys. 2019, 150, 114704. [CrossRef] [PubMed]

12. Busson, B.; Farhat, M.; Nini Teunda, P.J.; Roy, S.; Jarisz, T.; Hore, D.K. All-experimental analysis of doubly resonant sum-frequency generation spectra: Application to aggregated rhodamine films. J. Chem. Phys. 2021, 154, 224704. [CrossRef] [PubMed]

13. Peremans, A.; Caudano, Y.; Thiry, P.A.; Dumas, P.; Zhang, W.Q.; Le Rille, A.; Tadjeddine, A. Electronic Tuning of Dynamical Charge Transfer at an Interface: K Doping of C60/Ag(111). Phys. Rev. Lett. 1997, 78, 2999-3002. [CrossRef]

14. Caudano, Y.; Silien, C.; Humbert, C.; Dreesen, L.; Mani, A.A.; Peremans, A.; Thiry, P.A. Electron-phonon couplings at C60 interfaces: A case study by two-color, infrared-visible sum-frequency generation spectroscopy. J. Electron. Spectros. Relat. Phenom. 2003, 129, 139-147. [CrossRef]

15. Elsenbeck, D.; Das, S.K.; Velarde, L. Substrate influence on the interlayer electron-phonon couplings in fullerene films probed with doubly-resonant SFG spectroscopy. Phys. Chem. Chem. Phys. 2017, 19, 18519-18528. [CrossRef]

16. Chou, K.C.; Westerberg, S.; Shen, Y.R.; Ross, P.N.; Somorjai, G.A. Probing the charge-transfer state of CO on Pt(111) by twodimensional infrared-visible sum frequency generation spectroscopy. Phys. Rev. B Condens. Matter Mater. Phys. 2004, 69, 1-4. [CrossRef]

17. Busson, B.; Dalstein, L. Sum-Frequency Spectroscopy Amplified by Plasmonics: The Small Particle Case. J. Phys. Chem. C 2019, 123, 26597-26607. [CrossRef]

18. Dalstein, L.; Ben Haddada, M.; Barbillon, G.; Humbert, C.; Tadjeddine, A.; Boujday, S.; Busson, B. Revealing the Interplay between Adsorbed Molecular Layers and Gold Nanoparticles by Linear and Nonlinear Optical Properties. J. Phys. Chem. C 2015, 119, 17146-17155. [CrossRef]

19. Linke, M.; Hille, M.; Lackner, M.; Schumacher, L.; Schlücker, S.; Hasselbrink, E. Plasmonic Effects of Au Nanoparticles on the Vibrational Sum Frequency Spectrum of 4-Nitrothiophenol. J. Phys. Chem. C 2019, 123, 24234-24242. [CrossRef]

20. Kawai, T.; Neivandt, D.J.; Davies, P.B. Sum frequency generation on surfactant-coated gold nanoparticles. J. Am. Chem. Soc. 2000, 122, 12031-12032. [CrossRef]

21. Tourillon, G.; Dreesen, L.; Volcke, C.; Sartenaer, Y.; Thiry, P.A.; Peremans, A. Total internal reflection sum-frequency generation spectroscopy and dense gold nanoparticles monolayer: A route for probing adsorbed molecules. Nanotechnology 2007, $18,415301$. [CrossRef]

22. Tourillon, G.; Dreesen, L.; Volcke, C.; Sartenaer, Y.; Thiry, P.A.; Peremans, A. Close-packed array of gold nanoparticles and sum frequency generation spectroscopy in total internal reflection: A platform for studying biomolecules and biosensors. J. Mater. Sci. 2009, 44, 6805-6810. [CrossRef] 
23. Weeraman, C.; Yatawara, A.K.; Bordenyuk, A.N.; Benderskii, A.V. Effect of nanoscale geometry on molecular conformation: Vibrational sum-frequency generation of alkanethiols on gold nanoparticles. J. Am. Chem. Soc. 2006, 128, 14244-14245. [CrossRef]

24. Bordenyuk, A.N.; Weeraman, C.; Yatawara, A.; Jayathilake, H.D.; Stiopkin, I.; Liu, Y.; Benderskii, A.V. Vibrational sum frequency generation spectroscopy of dodecanethiol on metal nanoparticles. J. Phys. Chem. C 2007, 111, 8925-8933. [CrossRef]

25. Alyabyeva, N.; Ouvrard, A.; Zakaria, A.M.; Bourguignon, B. Probing Nanoparticle Geometry down to Subnanometer Size: The Benefits of Vibrational Spectroscopy. J. Phys. Chem. Lett. 2019, 10, 624-629. [CrossRef]

26. Molinaro, C.; Cecchet, F. Label-free, quantitative and sensitive detection of nanoparticle/membrane interactions through the optical response of water. Sens. Actuators B Chem. 2019, 289, 169-174. [CrossRef]

27. Humbert, C.; Noblet, T. A unified mathematical formalism for first to third order dielectric response of matter: Application to surface-specific two-colour vibrational optical spectroscopy. Symmetry 2021, 13, 153. [CrossRef]

28. Raschke, M.B.; Hayashi, M.; Lin, S.H.; Shen, Y.R. Doubly-resonant sum-frequency generation spectroscopy for surface studies Chem. Phys. Lett. 2002, 359, 367-372. [CrossRef]

29. Dreesen, L.; Humbert, C.; Celebi, M.; Lemaire, J.J.; Mani, A.A.; Thiry, P.A.; Peremans, A. Influence of the metal electronic properties on the sum-frequency generation spectra of dodecanethiol self-assembled monolayers on $\mathrm{Pt}(111), \mathrm{Ag}(111)$ and $\mathrm{Au}$ (111) single crystals. Appl. Phys. B Lasers Opt. 2002, 74, 621-625. [CrossRef]

30. Carron, K.T.; Hurley, L.G. Axial and azimuthal angle determination with surface-enhanced Raman spectroscopy: Thiophenol on copper, silver, and gold metal surfaces. J. Phys. Chem. 1991, 95, 9979-9984. [CrossRef]

31. Feugmo, C.G.T.; Liégeois, V. Analyzing the vibrational signatures of thiophenol adsorbed on small gold clusters by DFT calculations. ChemPhysChem 2013, 14, 1633-1645. [CrossRef]

32. Hosseinpour, S.; Roeters, S.J.; Bonn, M.; Peukert, W.; Woutersen, S.; Weidner, T. Structure and Dynamics of Interfacial Peptides and Proteins from Vibrational Sum-Frequency Generation Spectroscopy. Chem. Rev. 2020, 120, 3420-3465. [CrossRef] [PubMed]

33. Braun, R.; Casson, B.D.; Bain, C.D.; Van Der Ham, E.W.M.; Vrehen, Q.H.F.; Eliel, E.R.; Briggs, A.M.; Davies, P.B. Sum-frequency generation from thiophenol on silver in the mid and far-IR. J. Chem. Phys. 1999, 110, 4634-4640. [CrossRef]

34. Bozzini, B.; D’Urzo, L.; Mele, C.; Busson, B.; Humbert, C.; Tadjeddine, A. Doubly resonant sum frequency generation spectroscopy of adsorbates at an electrochemical interface. J. Phys. Chem. C 2008, 112, 11791-11795. [CrossRef]

35. Bozzini, B.; Abyaneh, M.K.; Busson, B.; Pietro De Gaudenzi, G.; Gregoratti, L.; Humbert, C.; Amati, M.; Mele, C.; Tadjeddine, A. supported Pt-Part III: Monitoring of electrodeposited-Pt catalyst ageing by in situ Fourier transform infrared spectroscopy, in situ sum frequency generation spectroscopy and ex situ photoelectron spectromicroscopy. J. Power Sources 2016, 231, 6-17. [CrossRef]

36. Kiessling, R.; Tong, Y.; Giles, A.J.; Gewinner, S.; Schöllkopf, W.; Caldwell, J.D.; Wolf, M.; Paarmann, A. Surface Phonon Polariton Resonance Imaging Using Long-Wave Infrared-Visible Sum-Frequency Generation Microscopy. ACS Photonics 2019, 6, 3017-3023. [CrossRef]

37. Ortega, J.M.; Glotin, F.; Prazeres, R. Extension in far-infrared of the CLIO free-electron laser. Infrared Phys. Technol. 2006, 49, 133-138. [CrossRef]

38. Pluchery, O.; Humbert, C.; Valamanesh, M.; Lacaze, E.; Busson, B. Enhanced detection of thiophenol adsorbed on gold nanoparticles by SFG and DFG nonlinear optical spectroscopy. Phys. Chem. Chem. Phys. 2009, 11, 7729-7737. [CrossRef]

39. Humbert, C.; Pluchery, O.; Lacaze, E.; Tadjeddine, A.; Busson, B. A multiscale description of molecular adsorption on gold nanoparticles by nonlinear optical spectroscopy. Phys. Chem. Chem. Phys. 2012, 14, 280-289. [CrossRef]

40. Humbert, C.; Pluchery, O.; Lacaze, E.; Tadjeddine, A.; Busson, B. Optical spectroscopy of functionalized gold nanoparticles assemblies as a function of the surface coverage. Gold Bull. 2013, 46, 299-309. [CrossRef]

41. Bain, C.D. Sum-frequency Vibrational Spectroscopy of the Solid/Liquid Interface. J. Chem. Soc. Faraday Trans. 1995, 91, 1281-1296. [CrossRef]

42. Eliel, E.R.; van der Ham, E.W.M.; Vrehen, Q.H.F. Enhancing the yield in surface sum-frequency generation by the use of surface polaritons. Appl. Phys. B Lasers Opt. 1999, 68, 349-353. [CrossRef]

43. van der Ham, E.W.M.; Vrehen, Q.H.F.; Eliel, E.R. Self-dispersive sum-frequency generation at interfaces. Opt. Lett. 1996, 21, 1448-1450. [CrossRef] [PubMed]

44. Mani, A.A.; Dreesen, L.; Humbert, C.; Hollander, P.; Caudano, Y.; Thiry, P.A.; Peremans, A. Development of a two-color picosecond optical parametric oscillator, pumped by a Nd:YAG laser mode locked using a nonlinear mirror, for doubly-resonant sum frequency generation spectroscopy. Surf. Sci. 2002, 502-503, 261-267. [CrossRef]

45. Grabar, K.C.; Hommer, M.B.; Natan, M.J.; Freeman, R.G. Preparation and Characterization of Au Colloid Monolayers. Anal. Chem. 1995, 67, 735-743. [CrossRef]

46. Su, K.H.; Wei, Q.H.; Zhang, X.; Mock, J.J.; Smith, D.R.; Schultz, S. Interparticle coupling effects on plasmon resonances of nanogold particles. Nano Lett. 2003, 3, 1087-1090. [CrossRef]

47. Funston, A.M.; Novo, C.; Davis, T.J.; Mulvaney, P. Plasmon coupling of gold nanorods at short distances and in different geometries. Nano Lett. 2009, 9, 1651-1658. [CrossRef] [PubMed]

48. Pellas, V.; Hu, D.; Mazouzi, Y.; Mimoun, Y.; Blanchard, J.; Guibert, C.; Salmain, M.; Boujday, S. Gold Nanorods for LSPR Biosensing: Synthesis, Coating by Silica, and Bioanalytical Applications. Biosensors 2020, 10, 146. [CrossRef] [PubMed]

49. Bossard-Giannesini, L.; Cruguel, H.; Lacaze, E.; Pluchery, O. Plasmonic properties of gold nanoparticles on silicon substrates: Understanding Fano-like spectra observed in reflection. Appl. Phys. Lett. 2016, 109, 111901. [CrossRef] 
50. Noblet, T.; Busson, B.; Humbert, C. Diagrammatic theory of linear and nonlinear optics for composite systems. Phys. Rev. A 2021, 104, 063504. [CrossRef]

51. Dalstein, L.; Revel, A.; Humbert, C.; Busson, B. Nonlinear optical response of a gold surface in the visible range: A study by two-color sum-frequency generation spectroscopy. I. Experimental determination. J. Chem. Phys. 2018, 148. [CrossRef] [PubMed]

52. Busson, B.; Dalstein, L. Nonlinear optical response of a gold surface in the visible range: A study by two-color sum-frequency generation spectroscopy. II. Model for metal nonlinear susceptibility. J. Chem. Phys. 2018, 149, 034701. [CrossRef] [PubMed]

53. Busson, B.; Dalstein, L. Nonlinear optical response of a gold surface in the visible range: A study by two-color sum-frequency generation spectroscopy. III. Simulations of the experimental SFG intensities. J. Chem. Phys. 2018, 149, 154701. [CrossRef] [PubMed] 